\title{
Procollagen IIC-peptide as a marker for assessing mechanical risk factors of knee osteoarthritis: effect of obesity and varus alignment
}

\author{
T Kobayashi, Y Yoshihara, H Yamada, K Fujikawa
}

\begin{abstract}
Objective-To ascertain by cross sectional examination whether the concentration of procollagen IIC-peptide in joint fluid significantly correlates with mechanical risk factors of knee osteoarthritis (OA), such as obesity (body mass index) and varus alignment (lateral femorotibial angle).

Methods-The concentrations of procollagen IIC-propeptide in synovial fluid were measured by a sandwich enzyme immunoassay of 65 patients with the same radiological stage of primary knee OA-that is, Ahlbäk stage I. The relations between procollagen IIC-peptide and body mass index and lateral femorotibial angle were examined using simple regression analysis and multiple regression analysis.
\end{abstract}

Results-Significant positive correlations were found between procollagen IICpropeptide concentrations and body mass index $(r=0.479, p<0.0001)$, and between procollagen IIC-propeptide concentrations and lateral femorotibial angle $(r=0.375, p=0.0021)$. Significant correlations were also found by multiple regression analysis. The multiple correlation coefficient of body mass index and femorotibial lateral angle to the procollagen IICpropeptide concentrations was 0.547 $(\mathbf{p}<0.0001)$.

Conclusions-The findings suggest that synthesis of type II collagen by chondrocytes is enhanced by abnormal mechanical stress, in this case obesity and varus alignment. It is concluded that procollagen IIC-propeptide concentrations in joint fluid are a useful marker of early OA. (Ann Rheum Dis 2000;59:982-984)

Assessment of the condition of articular cartilage in osteoarthritic knee joints is important for early diagnosis and evaluation of the effects of treatment. Although radiography and magnetic resonance imaging allow assessment of the degree of bone and cartilage attrition morphologically, joint fluid molecular markers serve as a method of metabolic assessment. As procollagen IIC-peptide is a marker of type II collagen synthesis, it is expected to be useful in monitoring the condition of osteoarthritic cartilage. $^{1-3}$ We therefore conducted cross sectional examinations to ascertain whether the concentration of procollagen IIC-peptide in joint fluid is significantly correlated with mechanical risk factors of knee osteoarthritis (OA), such as obesity and varus alignment.

\section{Methods}

Sixty five patients with the same radiological stage of primary knee OA-that is, Ahlbäk ${ }^{4}$ stage I, were examined. All patients were diagnosed according to the clinical and radiographic criteria of idiopathic knee OA provided by the American Rheumatism Association. ${ }^{5}$ Their average age was 64 (SD 11) years. Only eight of the 65 patients were men.

Joint fluid samples were aseptically obtained from the patients' knee joints. The fluids were centrifuged at $10000 \mathrm{rpm}$ for 20 minutes to remove cell and tissue debris, and the supernatants were stored at $-80^{\circ} \mathrm{C}$. Procollagen IIC-peptide concentrations in the joint fluid were determined by a one step sandwich enzyme immunoassay as previously described. ${ }^{2}$ The assay was linear between 0.2 and $20 \mathrm{ng} / \mathrm{ml}$. Intra- and interassay variability was $4-5 \%$.

The following variables were assessed: the relation between joint fluid procollagen IICpropeptide concentrations and obesity/varus alignment. Obesity was assessed by the body mass index (BMI). ${ }^{6}$ This is the ratio of weight in kilograms to height in metres squared-the index of relative weight named by Keys, which he considered preferable to other indices of relative weight. Among Japanese people a BMI between 19.8 and 24.2 is normal, $24.2-26.4$ is considered overweight, and more than 26.4 indicates obesity. ${ }^{7}$ Varus alignment was assessed by determination of the femorotibial lateral angle (FTA) by leg radiography carried out while the patient was standing on one leg. Tamaki and Koga conducted prospective field research among Japanese people ${ }^{8}$ and reported that in $203 / 820$ cases no osteoarthritic change to the knee joints according to the $x$ ray results had taken place either before or after seven years' follow up. The mean FTA was 173.9 (3.1) degrees and the mean (SD) age 55.4 (5.4) years. 
Simple regression analyses were carried out to assess the correlation between procollagen IIC-propeptide concentrations and BMI, and between procollagen IIC-propeptide concentrations and FTA. Multiple regression analysis was also performed. In particular we sought to establish whether procollagen IIC-propeptide concentrations correlate with the linear addition of BMI and FTA $(a \times$ BMI $+b \times$ FTA $)$. $p$ Values $<0.05$ were considered significant.

\section{Results}

The mean BMI was 24.4 (4.0) (range 14.836.9). This mean lies within the range denoting overweight among Japanese people. The mean FTA was 178.5 (5.8) degrees (range 170-192). This mean was slightly larger than the mean FTA of Tamaki's normal group. Because the correlation between BMI and FTA was not

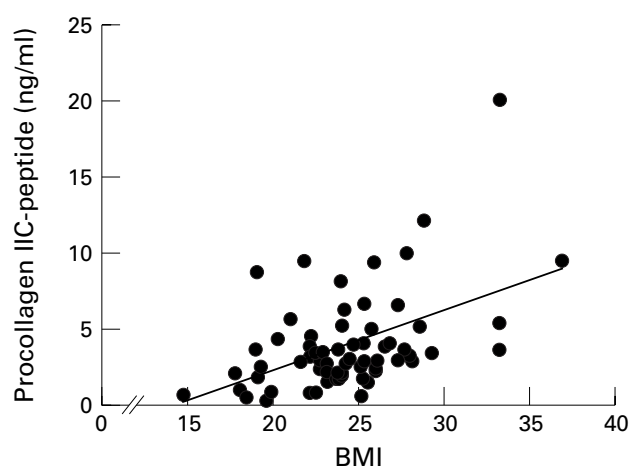

Figure 1 Correlation between procollagen IIC-peptide concentrations and body mass index (BMI). Procollagen IIC-propeptide concentrations in joint fluid were measured by enzyme immunoassay. Significant correlations were found between procollagen IIC-propeptide concentrations in joint fluid and BMI in 65 patients with primary knee osteoarthritis, Ahlbäk stage I $(r=0.479, p<0.0001)$.

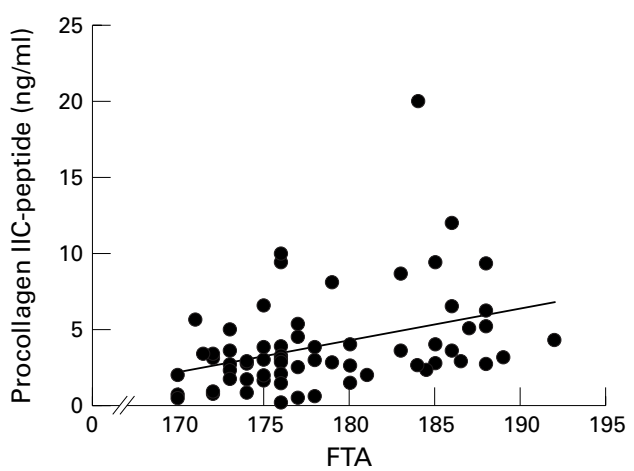

Figure 2 Correlation between procollagen IIC-peptide concentrations and lateral femorotibial angle (FTA).

Significant correlations were also found between procollagen IIC-propeptide concentrations in joint fluid and the FTA in 65 patients with primary knee osteoarthritis, Ahlbäk stage I $(r=0.375, p=0.0021)$.

Table 1 Results of multiple regression analysis among procollagen IIC-propeptide concentration, body mass index (BMI), and femorotibial lateral angle (FTA)

\begin{tabular}{lcrl}
\hline & Std Error & $t$ Value & $p$ Value \\
\hline BMI & 0.089 & 3.751 & 0.0004 \\
FTA & 0.062 & 2.483 & 0.0157 \\
Constant & 10.654 & -2.948 & 0.0045
\end{tabular}

Procollagen IIC-propeptide concentration $(\mathrm{ng} / \mathrm{ml})=0.355 \times$ $\mathrm{BMI}+0.153 \times \mathrm{FTA}-31.405$. significant $(r=0.249, \mathrm{p}=0.682)$, biomechanical BMI and FTA are considered independent mechanical factors.

The mean concentration of procollagen IICpropeptide in the joint fluid was 4.0 (3.2) $\mathrm{ng} / \mathrm{ml}$. This value was significantly higher than that previously determined by the same method using knee joint fluid from healthy volunteers. ${ }^{9}$

Significant positive correlations were found between procollagen IIC-propeptide concentrations and BMI $(r=0.479, \mathrm{p}<0.0001$, fig 1$)$, and between procollagen IIC-propeptide levels and FTA ( $r=0.375, p=0.0021$, fig 2). Significant correlations were also found by multiple regression analysis (table 1). The multiple correlation coefficient of the procollagen IICpropeptide concentrations to $(0.355 \times \mathrm{BMI}+$ $0.153 \times$ FTA-31.405) was $0.547(\mathrm{p}<0.0001)$.

\section{Discussion}

Procollagen IIC-propeptide is a noncollagenous carboxypeptide extension of type II procollagen molecules. During the formation of type II collagen fibrils, this extension is removed and released from type II procollagen molecules, implying that procollagen IICpropeptide release into joint fluid reflects type II collagen synthesis by chondrocytes. This is supported by the findings of Nelson's report. ${ }^{10}$ He found that the synthesis of type II collagen in the cartilage correlated with the content of procollagen IIC-propeptide in both the cartilage and the culture medium in vitro using human and bovine cartilage. He also reported that the content of procollagen IIC-peptide in articular cartilage is highest in fetal cartilage, being reduced almost threefold at birth and reaching low levels in normal adult cartilage; but in human OA cartilage it was significantly raised. ${ }^{10}$ In osteoarthritic knee joints the concentrations of procollagen IIC-propeptide in the joint fluids were found to be higher than in the joint fluids of healthy volunteers. ${ }^{9}$ We therefore examined whether procollagen IICpropeptide concentrations in primary knee OA are correlated with risk factors.

We limited this investigation to early stage knee OA only-namely, to Ahlbäk stage I knees. Because the articular cartilage is worn out in knees with advanced OA, the variability of the condition of the articular cartilage is likely to affect adversely an investigation of whether mechanical factors correlate with procollagen IIC-propeptide concentrations in joint fluid.

A variety of risk factors have been reported for primary OA, such as body weight, ${ }^{11}$ sex, ${ }^{12}$ working environment, ${ }^{13}$ and heredity. ${ }^{14}$ All these factors may influence type II collagen synthesis by chondrocytes. Felson, in his epidemiological analysis based on the Framingham study, ${ }^{11}$ reported that obesity as a mechanical risk factor was closely related to the incidence of knee OA. This was supported by our formerly reported research using joint fluid procollagen IIC-propeptide concentrations. ${ }^{1}$ We consider that varus alignment is another independent mechanical risk factor of $\mathrm{OA}$ because as the severity of the varus alignment 
of the knee joint increases the more the mechanical stress caused by body weight concentrates on the medial compartment of the knee joint, even if the body weight is the same.

Our findings suggest that synthesis of type II collagen by chondrocytes is enhanced by abnormal mechanical stress such as obesity and varus alignment as both were significantly positively correlated with procollagen IICpropeptide concentrations. We conclude that procollagen IIC-propeptide concentrations in joint fluid are a useful marker of early OA and reflect mechanical risk factors. The authors thank Dr K Ito of Teijin Co Ltd, for his coopera-
tion in measuring the procollagen IIC-propeptide. This research tion in measuring the procollagen IIC-propeptide. This research
is supported by the Scientific Research for Health and Welfare Programs of the Ministry of Health and Welfare of Japan.

1 Kobayashi T, Yoshihara Y, Samura A, Yamada H, Shimmei M, Lohmander LS, et al. Synovial fluid concentrations of the C-propeptide of type II collagen correlate with body mass index in primary knee osteoarthritis. Ann Rheum Dis mass index in prim

2 Shimmei M, Ito K, Matsuyama S, Yoshihara Y, Matsuzawa $\mathrm{K}$. Joint fluid carboxy-terminal type II procollagen peptide as a marker of cartilage collagen biosynthesis. Osteoarthritis Cartilage 1993;1:121-8.

3 Shimmei M, Kobayashi T, Yoshihara Y, Samura A Significance of the levels of carboxy-terminal type II procollagen peptide, chondroitin sulfate isomers, tissue inhibitor of metalloproteinases, and metalloproteinases in osteoarthritis joint fluid. J Rheumatol 1995;22(suppl):4381 .

4 Ahlbäck S. Osteoarthrosis of the knee-a radiographic investigation. Acta Radiol Suppl 1968;277:1-72.

5 Altman R, Asch E, Bloch D. Development of criteria for the classification and reporting of osteoarthritis. Arthritis Rheum 1986;29:1039-49.

6 Keys A, Fidanza F, Karvonen MJ, Kimura N, Taylor HL. Indices of relative weight and obesity. Journal of Chronic Diseases 1972;25:329-43.

7 Inoue S, Oosaka T, Tsuji M, Saitou N. Table of standard Inoue S, Oosaka T, Tsuji M, Saitou N. Table of stand
body weight. Nippon Rinsho 1998;56(suppl 1):781-8.

8 Tamaki M, Koga Y. Osteoarthritis of the knee joint: a field study. Journal of the Japanese Orthopaedic Association 1994;68:737-50.

9 Lohmander LS, Yoshihara Y, Roos H, Kobayashi T, Yamada $\mathrm{H}$, Shinmei M. Procollagen II C-propeptide in joint fluid, changes in concentration with age, time after joint injury and osteoarthritis. J Rheumatol 1996;23:1765-9.

10 Nelson F, Dahlberg L, Laverty S, Reiner A, Pidoux I, Ionescu $M$, et al. Evidence for altered synthesis of type II collagen in patients with osteoarthritis. J Clin Invest 1998; 102:2115-25.

11 Felson DT, Anderson JJ, Naimark A, Walker AM, Meenan RF. Obesity and knee osteoarthritis, the Framingham study. Ann Intern Med 1988;109:18-24.

12 Spector TD, Perry LA, Jubb RW. Endogenous sex steroid levels in women with generalised osteoarthritis. Clin Rheumatol 1991;10:316-19.

13 Felson DT, Hannan MT, Naimark A, Berkeley J, Gordon G, Wilson PWF, et al. Occupational physical demands, knee bending, and knee osteoarthritis: results from the Framingham study. J Rheumatol 1991;18:1587-92.

14 Pun YL, Moskowitz RW, Lie S, Sundstrom WR, Block SR, McEwen C, et al. Clinical correlations of osteoarthritis associated with a single-base mutation (arginine ${ }^{519}$ to cysteine) in type II procollagen gene: a newly defined pathogenesis. Arthritis Rheum 1994;37:264-9. 